\title{
Implementation of a Facility Based County Surveillance System Using Epi Info
}

\author{
Samantha Spoto*1, Michael Wiese ${ }^{1}$ and Michelle Lyman ${ }^{2,1}$ \\ 'Epidemiology, Florida Department of Health in Hillsborough County, Tampa, FL, USA; ${ }^{2}$ University of South Florida College of Public \\ Health, Tampa, FL, USA
}

\section{Objective}

The Florida Department of Health in Hillsborough County (DOHHillsborough) routinely reviews the ESSENCE-FL system to assess syndromic trends in emergency department (ED) and urgent care data (UCC). Collection of this type of symptom data from long term care facilities (LTCFs) and child care centers is of interest in order to better understand how these illness patterns present in vulnerable populations outside of the EDs.

\section{Introduction}

Surveillance in nursing homes (Enserink et al., 2011) and day care facilities (Enserink et al., 2012) has been conducted in the Netherlands, but is not commonly practiced in the United States (Buehler et al., 2008). Outbreaks of illnesses within these facilities are required to be reported to the Epidemiology Program, however a small fraction of outbreaks reported come from LTCFs. Without regular communication between LTCFs and the Epidemiology Program, it is likely that many outbreaks are going unreported due to lack of awareness of the reporting requirements by facility staff. To better understand the prevalence of illness in LTCFs and improve communication between LTCFs and DOH-Hillsborough a weekly surveillance survey was created using Epi Info web survey.

\section{Methods}

The online facility search tool from the Agency for Healthcare Administration (AHCA) was used to query assisted living facilities and nursing homes in Hillsborough County in July 2017. The information provided included the number of beds a facility is licensed to have. Interest in participation was solicited from larger LTCFs within the county in August 2017 and 23 facilities volunteered to receive weekly surveys, with a total volume of 3,276 beds. A form was created in Epi Info to capture weekly information per facility of the number of residents and staff with new onset of various symptoms. Symptom groups include GI, rash, respiratory, and those with respiratory symptoms who also have a fever (to assess influenza-like illness); number of positive flu tests for the week is also asked. Starting with week 38, an email has been sent once a week to participating facilities with a link to the Epi Info web survey (Figure 1) and instructions to fill out the information for the previous week.

\section{Results}

To date, 12 weeks of information has successfully been captured in Epi Info and transferred to Microsoft Excel for graphical visualization of percentage of residents/staff reported each week in the county with new onset of the above symptoms. Low levels of illness $(<6 \% /$ week of total reported residents/staff) have been reported for various syndromes each week. Over the 12 week period an average of 3.9 facilities submit data per week, with a total of 10 of 23 facilities participating at least once. In week 42 phone calls were made to faclities that had not submitted any responses in an attempt to elicit more participation and troubleshoot any problems faclities may have encountered. Prior to week 42, an average of 3.2 facilties reported per week. After reminder phone calls were conducted, the average number of responses for weeks $42-48$ was 4.4 with the highest in week 42 ( 6 responses). Starting in week 42 the survey has also been implemented for 15 child care facilities, with four participating over the seven weeks with an average of 2.1 responses per week.

\section{Conclusions}

Since implementation, the main limitation with the data collection is lack of regular participation from facilities. The current goal of the project is to increase the number of regular responses from both LTCFs and child care facilities. The phone calls made in week 42 increased the response rate for LTCFs, particularly for that week. Preliminary results from the first 12 weeks of data indicate that using Epi Info web survey as a syndromic surveillance tool for local facilities has potential if regular participation can be acheived.

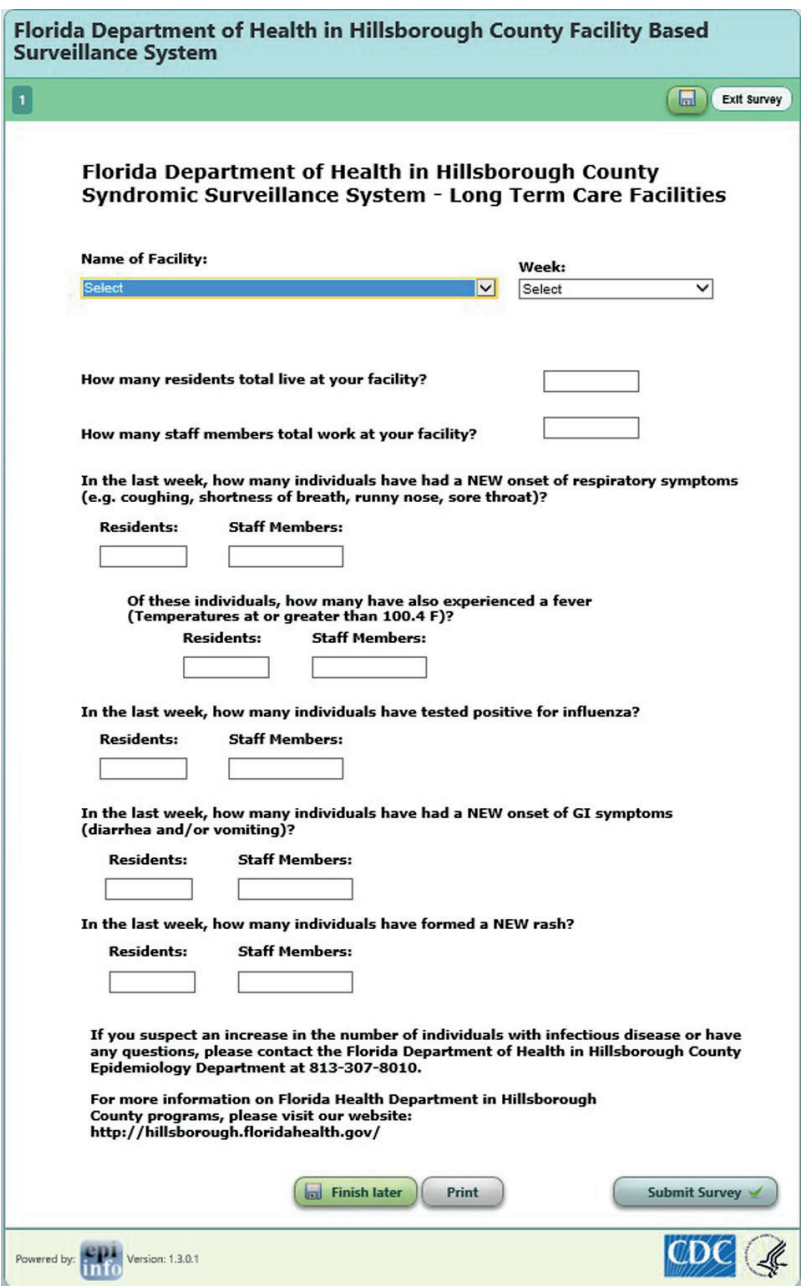

Figure 1. Screenshot of the Epi Info web survey for long term care facilties, to be completed on a weekly basis. 


\section{ISDS 2018 Conference Abstracts}

\section{Keywords}

Syndromic Surveillance; Long Term Care Facility; Epi Info

\section{References}

Buehler, J., Sonricker, A., Paladini, M., Soper, P., Mostashari, F. (2008), Syndromic Surveillance Practice in the United States: Findings from a Survey of State, Territorial, and Selected Local Health Departments. Advances in Disease Surveillance, 6:3.

Enserink, R., Meijer, A., Dijkstra, F., van Benthem, B., van der Steen, J. T., Haenen, A., van Delden, H., Cools, H., van der Sande, M., Veldman-Ariesen, M.-J. and on behalf of the Sentinel Surveillance Network on Infectious Diseases in Nursing Homes Study Group (2011), Absence of Influenza A(H1N1) During Seasonal and Pandemic Seasons in a Sentinel Nursing Home Surveillance Network in the Netherlands. J Am Geriatr Soc, 59: 2301-2305. doi:10.1111/ j.1532-5415.2011.03715.x

Enserink, R., Noel, H., Friesema, I., de Jager, C., Kooistra-Smid, A., Kortbeek, L., Duizer, E., van der Sande, M., Smit, H., van Pelt, W. (2012), The KIzSS network, a sentinel surveillance system for infectious diseases in day care centers: study protocol. BMC Infectious Diseases, 12:259. doi:10.1186/1471-2334-12-259

\section{*Samantha Spoto}

E-mail: samantha.spoto@flhealth.gov 